\title{
Transfert de gènes: système d'évaluation de l'activité pharmaco-toxicologique de petites molécules
}

\author{
F. Schweighoffer, I. Barlat, B. Tocqué
}

$\mathbf{L}$ 'introduction de gènes clonés dans des cellules d'eucaryotes supérieurs a permis d'accéder à la fonction précoce de protéines impliquées dans des voies de signalisation cellulaire diverses.

Les résultats observés par transfection transitoire dans le modèle cellulaire choisi doivent être reproductibles et cohérents avec les effets biologiques à long terme obtenus par expression stable de gènes ou par action de petites molécules modifiant l'expression de ceux-ci ou modulant l'activité des protéines correspondantes. Les exigences de reproductibilité et de cohérence posent le problème de la sensibilité et de l'efficacité des techniques utilisées pour l'introduction de gènes clonés dans des cellules d'eucaryotes supérieurs.

A côté des méthodes classiques au phosphate de calcium ou au DEAEDextran, assez limitées notamment pour l'étude de compétitions entre différents vecteurs, on a développé des techniques qui utilisent des agents lipidiques. Le DOTMA [1], par exemple, permet d'obtenir des liposomes qui capturent l'ADN à transfecter et qui vont pouvoir fusionner ensuite avec la membrane plasmique.

Le DOGS, développé plus récemment, permet une association étroite de deux chaînes aliphatiques avec l'ADN étudié grâce à une tête de spermine qui interagit avec le grand sillon de la double hélice. Contrairement aux lipo-

F. Schweighoffer, I. Barlat, B. Tocqué : Rhône-Poulenc Rorer SA, 13, quai JulesGuesde, BP 14, 94403 Vitry-sur-Seine Cedex, France. somes, l'action de l'agent de transfection ne consiste donc plus ici en une capture statistique de l'ADN et assure, dans les différents systèmes étudiés, une meilleure efficacité.

Nous avons utilisé les performances de ce moyen de transfection pour développer un test transitoire d'évaluation des fonctions des oncogènes ras. Les résultats ont non seulement confirmé ceux des autres systèmes d'évaluation, mais ont également permis de mettre en évidence des phénomènes moléculaires indétectables par les méthodes précédemment utilisées.

Les protéines Ras font partie d'une large famille de protéines fixant les nucléotides guanyliques. On peut identifier de telles protéines impliquées dans des processus cellulaires aussi variés que la réponse aux hormones et aux facteurs de croissance (protéines G-hétérotrimériques), la synthèse protéique (facteurs d'élongation), des processus de croissance, de différenciation cellulaire, de transport d'organites et de sécrétion (protéines $\mathrm{G}$ de petit poids moléculaire 20000 [3]. Cette famille des protéines $G$ de petit poids moléculaire comprend trois branches principales représentées par les protéines Ras elles-mêmes, les protéines Rho (Ras-homologous) et les protéines Rab (Ras from rat brain) [4].

Les gènes ras sont les proto-oncogènes les plus fréquemment retrouvés activés dans les tumeurs humaines $[5,6]$. Cette proportion qui peut atteindre plus de $95 \%$ dans certaines tumeurs est en augmentation avec la mise au point des techniques de détection par polymerase chain reaction (PCR). L'étude des fonctions de ces proto-oncogènes doit être un effort prioritaire pour la recherche en oncologie. Les protéines Ras évoluent entre un état actif lié au GTP et un état inactif lié au GDP [7]. Toutes les mutations activant le protooncogène affectent la structure de la protéine au niveau du site de liaison du nucléotide. Elles ont pour effet d'augmenter la proportion de formes GTP de la protéine [8]. Seules les formes actives, liées au GTP, sont capables d'interagir avec une protéine GAP, cette interaction nécessitant l'intégrité de "domaines effecteurs " [9]. Un débat important a pour enjeu de définir les fonctions de cette protéine GAP. Elle pourrait porter à la fois une fonction régulatrice (par augmentation de la vitesse d'hydrolyse du GTP sur la protéine Ras) et une fonction de signalisation [7].

In vivo, on ne connaît pas encore la nature de l'événement responsable de l'activation des protéines Ras, c'est-àdire de la transition p21 GDP $\rightarrow$ p21 GTP.

De telles activités ont été rapportées dans la littérature [10-12], mais l'identification et le clonage du gène n'ont pas encore été réalisés. En revanche, la protéine CDC 25 de Saccharomyces cerevisiae semble bien jouer ce rôle pour les protéines Ras de levure [13].

De plus, dans ces levures, les protéines Ras peuvent être fonctionnellement remplacées par des protéines de mammifères suggérant ainsi une communauté de structure entre CDC 25 et un facteur d'échange présent chez les mammifères [15].

Par complémentation des mutations létales de CDC 25, un autre gène de levure (SDC 25) a été identifié. La partie 3' de ce gène est capable d'assurer la fonction d'échange et, 
ainsi tronquée, confère à la levure un phénotype équivalent à celui obtenu après expression d'un oncogène ras [14].

Ce même gène, exprimé dans des cellules $\mathrm{CHO}$, provoque la charge en GTP des protéines Ras, assurant ainsi à nouveau dans la cellule un signal équivalent à celui obtenu après expression d'un oncogène ras [15].

Il existe peu d'événements cellulaires quantifiables et rapides décrivant l'activité de ces oncogènes et des protéines connexes.

L'un des moyens d'étudier les étapes de régulation évoquécs et de s'affranchir de l'établissement de cellules exprimant de manière stable des gènes impliqués (éventuellement létaux) est de transfecter, en parallèle, un gène rapporteur.

Puisque les protéines de la famille $\mathrm{Ras}$ interviennent dans la régulation de certains promoteurs, nous avons évalué lcur activité sur l'expression du gène $C A T$ placé sous contrôle du promotcur du LTR du HIV1.

Les résultats obtenus avec les oncogènes ras, certains de leurs mutants, un membre de la famille ras, rapla, ou l'extrémité C-terminale de SDC25 sont résumés dans le Tableau $I$.

Ces quelques données soulignent l'intérêt de tels essais d'expression transitoire. Tout d'abord, les résultats sur l'activation du gène rapporteur corroborent ccux obtenus après expression stable (formation de foyer de transformation) ou après introduction directe dans les cellules des protéines (stimulation de la synthèse d'ADN, maturation des ovocytes).

L'expression du gène rapportcur, élément de mesure précoce et rapide, semble donc représentative des événements cellulaires déclenchés par les oncogènes ras au même titre que des mesures représentant le résultat d'événements encore plus complexes.

De plus, ces essais permettent de mettre en évidence des événements moléculaires très discrets qui ne pourraient pas l'être par l'utilisation des autres techniques. Par exemple, la transactivation du LTR-HIV par rapla représente, en dehors des effets d'antagonisme de la transformation cellulaire (technique particulièrement lourde et

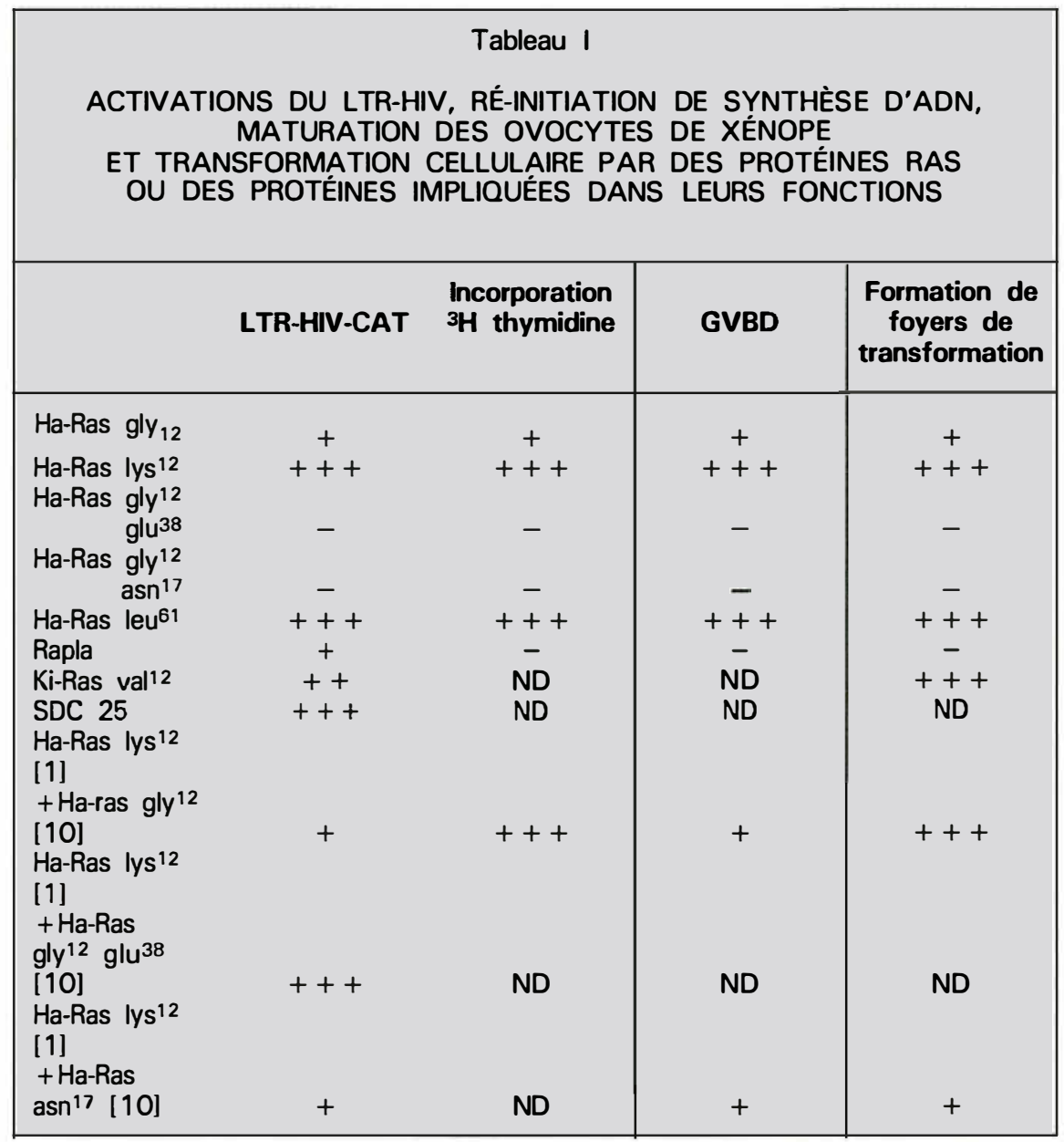

Le test de transactivation du LTR-HIV-CAT a été effectué selon [16], l'incorporation de thymidine après scraping (germinal vesical breakdown) des cellules NIH 3T3 selon [17], la maturation des ovocytes de xénope ou GVBD, suivant la technique décrite en [18], et la formation de foyers [8]. Dans les études de compétition par cotransfection, les plasmides compétitifs ont été ajoutés en excès de 10 fois [16]. ND : non dosé.

délicate à mettre en ouvre), le scul critère d'activité cellulaire de cette protéine.

De même, alors que dans les essais de transformation cellulaire ou de réinitiation de la synthèse d'ADN, il n'est évidemment pas possible de détecter une compétition entre une protéine Ras gly ${ }^{12}$ et son mutant activé Ras lys ${ }^{12}$, les transfections transitoires confirment les résultats d'injection dans les ovocytes de xénope. En l'occurrence, ces deux systèmes, qui traduisent des effets à court terme, permettent de suivre une compétition des deux formes de Ha-ras vis-à-vis d'un effecteur commun qui dépend de l'intégrité de leur zone effectrice.

Enfin, nous avons pu montrer que SDC 25, un activateur des protéines Ras, stimulait en effet la transactivation du gène test HIV-1-CAT. Ce résultat anticipe des études dans les autres systèmes expérimentaux. On peut naturellement suggérer que ces essais d'expression transitoire puissent être utilisés pour détecter et évaluer l'activité d'agents toxiques ou pharma cologiques suspectés d'agir sur les fonctions d'une protéine précise 


\section{Summary}

Genc transfer experiments : an efficient mean to cvaluate the pharmaco-toxicology of active compounds

The use of a very efficient lipidic agent for transfection of mammalian cells open new avenues for the evaluation of interaction between proteins at the molecular level that would not be detectable by other means. Expression of a given plasmid along with a reporter gene might facilitate evaluation of such interactions. We took advantage of such an assay to evaluate the cellular activity of combination of different proteins of the ras-family.

\section{Références}

1. Felgner LP, Gadek RT, Holm M, et al. Lipofection : a highly efficient, lipid media- ted DNA-transfection procedure. Proc Natl Acad Sci USA 1987 ; 84 : 7413-7. 2. Behr JP, Demeinex B, Loeffler JP, PereyMutul J. Efficient transfer into mammalian primary endocrine cells with lipopolyaminescoated DNA. Proc Natl Acad Sci USA 1989 ; $86: 6982-6$.

3. Bourne HR, Sanders DA, McCormick F. The GTPases superfamily: a conserved switch for diverse cell functions. Nature 1990 ; 348: 125-32.

4. Sanders DA. A guide to low molecular weight GTPases. Cell Growth Differ 1990 ; 1 : 251-8.

5. Bos JL. The ras gene family and human carcinogenesis. Mul Res $1988 ; 195$ : 255-71.

6 . Suarez HG. Activated oncogenes in human tumors. Anticancer Res $1989 ; 9$ : 1331-44.

7. McCormick F. Ras GTP ase activating protein : signal transmitter and signal terminator. Cell 1989 ; 56 : 5-8.

8. Barbacid. Ras genes. Ann Rev Biochem $1987 ; 56: 779-827$.

9. Calès C, Hancock JF, Marshall CJ, Hall A. The cytoplasmic protein GAP is implicated as the target for regulation by the ras gene product. Nature 1988 ; 332 : 548-51.

10. Wolfman A, Macara IG. A cytosolic protein catalyses the release of GDP from p $21^{\text {ras }}$. Science $1990 ; 248$ : 67-9.

11. West M, Kung H, Kamata T. A novel membrane factor stimulates guanine nucleotide exchange reaction of Ras protein. FEBS Lett 1990 ; 259 : 245-8.
12. Downward J, Rechl R, Wu L, Weinberg RA. Identification of a nucleotide exchange promoting activity of $21^{\text {ras }}$. Proc Natl Acad Sci USA 1990 ; 87 : 5998-6002.

13. Gibbs JB. Marshall MS. The ras oncogene : an important regulatory element in lower eucaryotic organisms, Mirob Rev 1989 ; $53: 171-85$.

14. Boy-Marcotte E, Damak F, Camonis J, Garreau H, Jacquet M. The C-terminal part of a gene partially homologous to $C D C 25$ gene suppresses the odc 25-5 mutation in Saccharomyces cerevisiae. Gene 1989; $77: 21-30$.

15. Rey I, Schweighoffer F, Barlat I, et al. The cooh-domain of the product of the Saccharomyces cerevisiae SCD25 gene elicits activation of $\mathrm{p}^{\text {ras }}$ protein in mammalian cells. Oncogene (sous presse).

16. Schweighoffer F, Rey I, Barlat I, Soubigou P, Mayaux JF, Tocqué B. Rap gene products mobilize a different metabolic pathway than $\mathrm{p} 21^{\text {ras }}$ proteins. Adv Sec Mess Phosph Res 1990 (Nishizuka Y, Indo M, Tanaka C, eds) Raven Press 1990 ; 53 : 329-34.

17. McNeil PL, Murphy RF, Lanni F, Lansing Taylor D. A method for incorporating macromolecules into adherent cells. J Cell Biol $1984 ; 98: 1556-64$.

18. Hirai S, Le Goasgogne C, Baulieu E. Introduction of geminal vesical breakdown in Xenopus laevis oocytes. Dev Biol 1984; 100 : 214-21. 